\title{
SCIENCE.-SuPPLEMENT.
}

FRIDAY, NOVEMBER 19, 1886.

\section{EXPERIMENTAL PSYCHOLOGY IN LEIPZIG}

THE period in the development of a science at which observation is supplemented by experimentation has long been recognized as one of critical importance. Moreover, if the nature of the science thus advanced seems to be such that the employment of the new instrument is followed by the positing of a more complete and scientific stand-point; if, in other words, the influence of the experimental stage is as valuable for theory as for practice, - the importance of this step is certainly increased. There are many men now living who could have witnessed the beginnings of this movement in psychology, and lived its life with their own. Notwithstanding the great enthusiasm with which this departure was hailed, - an enthusiasm which in its short career has experienced many ups and downs, the study has been taken up more largely as an avocation than as a serious life-work. Many scientists, mostly physicists or physiologists or alienists (Helmholtz, Mach, Hennig, Preyer), have taken up the limited portion of the subject in which they were most interested, and devoted themselves to it. The greatest advances of any have undoubtedly resulted from the labors of such men. On the other hand, the propounders of psychological systems have not been slow in incorporating the results and conceptions of the new movement into their doctrines, not always, it may be added, with a very congruous result. But there are many indications that an essential condition of the flourishing of scientific psychology is the existence of specialists devoted to its cause, with all the advantages, both material and intellectual, that their position in a first-class university can bring. Psychology is ready to emerge from the nomadic state; and, having given assurance of its permanency, it asks for a home, or rather for homes. The University of Leipzig, owing to the efforts of Professor Wundt, has been, perhaps, the foremost in answering this call. Many young men have gained an impetus for such work under his direction; and a quarterly Philosophische studien, devoted mainly to the publication of results of research in the Leipzig laboratory, was founded some years ago. The articles relating to experimental topics in the last two numbers of this journal ${ }^{1}$ will indicate the direction in which work is being done.

A very interesting study is that on the 'Memory for tone,' by Mr. H. K. Wolfe. The impetus to the research was given by the admirable study of the memory by Dr. Emminghaus, in which he counted the number of repetitions of a series of nonsense-syllables necessary to enable the hearer to repeat the series from memory at once or after a certain interval. He found, for example, that he could repeat seven such syllables when read to him but once; if there were twelve syllables, they would have to be repeated sixteen times, and if twenty-four syllables forty-four times before they were memorized. Mr. Wolfe very justly remarks that what is here understood by memory is the power to reproduce, and that there is a more simple and retentive form of memory, which consists in the power to recognize as familiar an object that has been presented to the senses before. A very common illustration of this is seen in the fact, that, on reading a book a second time, we recognize a great deal more of it than we could have told of it. So, too, we can recognize at least ten times as many shades of color as we can see in the imagination, can understand more words than are in our usual vocabulary ; and so on. It is this simpler form of memory that Mr. Wolfe studies. A series of nearly 300 vibrating metal tongues, giving the tones through five octa ves, from 32 to 1,024 vibrations, was at his disposal. These tongues gave tones differing by 2 vibrations only in the two lower octaves, and by 4 vibrations in the three higher octaves. In the first series of experiments a tone was selected, and, after sounding it for one second, a second tone was sounded, which was either the same as the first, or different from it by 4, 8, or 12 vibrations in different series. The person experimented upon was to answer whether the second tone was the same as the first, thus showing that he recognized it, or whether it was different, and, if so, whether it was higher or lower. Of course, the interval of time between the two tones was an important factor. The proportionate number of correct judgments, and the smallness of the difference of the vibration-rates of the two tones, would measure the accuracy of the tone memory. It appeared that one could tell more readily whether the two tones were alike than whether they were different,

1 Philosophische studien. Herausgegeben von WILHELM WUNDT. Band ili. hefte 3, 4. Leipzig, Engelmann, 1886. $8^{\circ}$. 
although in both cases the accuracy of the memory was remarkably good." When the tones were really equal, they were recognized as such, on the average, in from seventy-five to eighty per cent of all cases. In using tones differing by only 4 or 8 vibrations, though the difference was very often clearly perceived, the direction of it, whether higher or lower, was not always clear, and even in differences of 12 vibrations there was little confidence in one's judgment. This seems to be a peculiarity of auditory sensations: for in sight you can almost as readily say that a shade is lighter or darker than another as that it is different; you can almost as soon detect the direction in which a point is moving along the skin as you can detect the motion itself. But the main point is the effect of the time-interval between the tone and its reproduction. This was varied from 1 second to 30 seconds, or even to 60 seconds, or 120 seconds in some experiments. The general result is, that the longer the interval, the smaller the chances that the tone will be recognized; and this process of forgetting takes place at first very rapidly, and then more slowly. It is made probable that the interval must increase in a geometrical ratio to produce an arithmetical series of (approximately) equal degrees of forgetting ; i.e., the curve is logarithmic. This law is subject to considerable variations, one of which seems to be constant and is peculiar ; namely, there seems to be a rhythm in the memory itself, and, after falling, it recovers slightly, and then fades out again. Among other results were that the accuracy of the memory decreases as the pitch of the tone is lowered (within limits); that relatively high tones tend to be judged too high, and low ones too low, by unmusical ears ; that the effect of practice is at first marked, but soon diminishes as is its general law ; and that the recovering power of the ear is so great that fatigue has little effect. To prove the last proposition, experiments were made for one day from 8 A.M. to 7 P.M. (with ten minutes intermission).

A subject that has always received great attention at the Leipzig laboratory is the measurement of the time of psychic processes. These have been conveniently divided into three kinds : $1^{\circ}$, the reaction time, which is simply the time after the application of the sense-stimulus necessary for an individual to record the fact that he has received the sensation; $2^{\circ}$, the distinction or per-

1 Very unfortunately, Mr. Wolfe, in tabulating his results, has worked upon a false mathematical process, and has thus made it impossible to draw conclusions regarding the recognition of the fine intervals of tone. From the original records such conclusions could be drawn. I am thus forced, on this account, to speak only of the recognition of equality of tone, and even to make allowances in stating these. ception time, which is the additional time necessary for him to appreciate the nature of the sensation, e.g., whether a light was red or blue : $3^{\circ}$, the choice or will time, which is the additional time necessary to react in a certain way on the reception of a certain sensation, e.g., to press a key with the right hand when the red light appears, with the left hand or not at all for the blue light. Dr. J. M. Cattell, in a recent re investigation of a large part of the field, has brought to notice several new facts, and has improved the method in many respects. To insure himself against any variations in the working of his apparatus, Dr. Cattell devised a means of controlling it, an essential part of the device being the determination of the most suitable strength of current. for running the chronoscope. The time is recorded on a Hipp chronoscope, which, by the release of a magnet and the springing back of the same, records intervals of one one-thousandth of a second. A falling screen, at a point in its fall, suddenly reveals a card or color, if that is the sense-stimulus, or can convey a shock to the finger, etc., and at the same time releases the magnet of the chronoscope, and sets the hands of the clock in motion. The reaction of the observer is made either by closing a key connected with the chronoscope with his hand, or by speaking through a tube, which, like the hand-key, has the effect of instantly stopping the clock. One can then read on the chronoscope the interval of time between the two events. In this way it was found, as the result of 520 experiments on each of two observers extended over a period of six months, that the reaction time for daylight, reflected from a white surface, was quite constant, and was about .149 of a second (strictly, .151 of a second for one, .147 of a second for the other observer), it being immaterial whether the reaction was made with the right or the left hand. But it takes .030 of a second longer to record the reaction by moving the lips. It is usually considered that the state of the attention has most effect on the reaction time; but Dr. Cattell found that the disturbance caused by the ticking and ringing of metronomes with hell attachments affected the reaction very slightly indeed, and explains this divergence from the results of other experimenters, by the unusual amount of practice which he had in such experiments. In other words, the process was too automatic to be affected seriously by the attention. Again : if the attention be distracted by the mental operation of repeaterly adding 17 to a series of numbers, the time is more seriously lengthened ; and, if the observer makes a great effort to attend, the time can be slightly shortened. He also shows that this extreme state of attention can be main- 
tained for only about one second. The two observers show some individual variations, all of which indicate that the processes were more thoroughly reflex in Dr. Cattell's case than in that of his associate. It is argued that the cortex is not concerned in the reaction, and that perception and willing also have no part in it.

Passing to perception times, the observer is asked to react only if the card which the falling of the screen reveals has a white surface, and simply do nothing at all if it has a black surface. The additional time necessary to recognize the whiteness of the surface, and to send out the voluntary impulse, was for the one observer .061 of a second ; for the other, .095 of a second. This time Dr. Cattell thinks should be divided equally between the two operations, because in the simple reaction the closing of the key was entirely automatic. Of course, it no longer makes any difference how the reaction is made. It, however, takes a little longer, if, instead of a white surface, a colored surface is used, though the observer has simply to distinguish that it is not black. If the color is to be distinguishel, and in each case can be one of two colors, then the perception-and-will time was .100 and .110 of a second respectively for the two observers; if the color can be one of ten colors, .105 and .117 of a second. In other words, it takes about .0058 of a second longer to distinguish one of ten colors than one of two, but .033 of a second longer to say what the color is than to say it was not black. In a similar way, if two letters are to be distinguished from one another, instead of two colors, the time is lengthened by about .038 of a second. If a letter is to be distinguished out of ten letters, it matters greatly what the letter is. $\mathrm{E}$ is the most difficult to read. The order of difficulty of five letters experimented upon was M, A, Z, B, E. The perception time for short English words is somewhere about .142 of a second ; it is slightly longer for long words and for words in a foreign language. A very important point is, that the perception time for words is only slightly longer than for letters; thus showing that the former, not the latter, is the reading unit. Finally, if small pictures of such familiar objects as a tree, hand, etc., be used instead of words, the time is found to be shorter, and is about equal to that for seeing a color. A picture is thus a simpler, less abstract sign than a series of letters.

There remains the will or choice time. We have seen in the series of experiments just described that a will time has already entered. If we change the form of experiment so that if a red light appears the right hand is to react, and if a blue the left hand, the time is lengthened by .026 of a second. In the former case the same hand always reacted; now the motor impulse is to be sent in a certain direction. ${ }^{1}$

A very interesting form of the experiment consists in letting the different kinds of reaction be the names of the colors, letters, pictures, or words ; in other words, to measure the time necessary to read, i.e., to see and name them. Four-tenths of a second was thus found as the time necessary for reading a letter. Similarly the time necessary for reading a one-place number was found to be about .360 of a second, it taking only .033 of a second longer to read two-place numbers, and only .025 of a second longer for a three-place than a twoplace number. As regards words, it was found that it took longer to read (not including pronunciation) long than short, and foreign than vernacular words. To name a short word in one's native language requires .111 of a second, which is .050 of a second less time than it takes to name a letter ; thus showing, that, as we constantly read words and seldom letters, the association between the concept and the name is much closer. An interesting result as regards color is this: that, while it takes less time to perceive a color than a le'ter or word, it takes quite a long time (.343 of a second) to find the name of the color. The association between a color and its name is a loose one. The names of the more familiar colors were found in considerably less time. The average time for naming a picture is about equal to that for naming a familiar color. Familiarity is again an important factor : it took least time to name a ' hat,' longest to name a 'teapot.' By way of summary, the following table will be found convenient. ${ }^{2}$

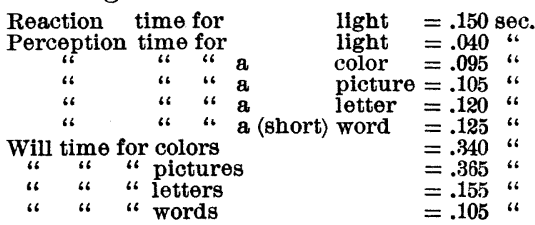

There have thus been recorded the times of some of the simpler mental activities, and the understanding of the higher psychic processes has thus been made more easy. The times of these processes Dr. Cattell supposes will become shorter in the course of evolution " and we will live so much the"longer in the same number of years."

Finally, a very painstaking research by Dr. Alfred Lehmann deserves mention. His object

1 The perception process is also slightly but not materially different.

2 The final section of the paper is devoted to showing that extreme attention can shorten central operations; that the effect of practice is flrst marked, but soon reaches its limit (as automatism sets in); that fatigue is not as readily brought on, and is not as disturbing a cause as is usually supposed. 
was to decide whether the method of the 'mean gradations,' is applicable to the sensations of brightness. This method consists in presenting two disks composed of different proportions of black and white (and thus, when rapidly revolved, showing different shades of gray), and asking the observer to regulate the amount of black and white in a third disk until it was just as much darker than the one as it was lighter than the other : i.e., to find a gray of a mean intensity. Call the intensity of the darker one $x$ and of the lighter one $y$, then the arithmetical mean would be $\frac{x+y}{2}$. But if Weber's law (which says, that to produce equal differences of sensation, the difference in the stimuli vary proportionately to the stimulus already present) is true, then $v x y$ would be the intensity that appears to be mean; i.e., $x: \vee v y:: \vee x y: y$. Dr. Lehmann's study is devoted to discovering all the sources of error in such an experiment. The order of the disks, whether the variable disk should be between the two or not; the direction and kind of illumination ; the order of experimenting ; and, beyond all, the effect of contrast with the back-ground against which the disks were seen, - were all taken into account. Dr. Lehmann succeeded in measuring quantitatively the effect of contrast (a rery valuable result), applied a method of eliminating its effect, but finally comes to the purely negative conclusion that the question of the validity of Weber's law is not favored or refuted by his experiments. A real test still remains to be made. His most valuable result is the study of the great effect of contrast in all such work.

JOSEPH JASTROW.

\section{PROCEEDINGS OF THE ENGLISH SOCIETY FOR PSYCHICAL RESEARCH.}

To allay at once any feelings of expectancy (which some readers may share with the writer whenever the green cover of a new number of these 'Proceedings' is caught sight of) it may be well to say that nothing unusually wonderful is therein revealed. A considerable portion of the number is devoted to the 'physical phenomena' connected with spiritualism. Fortunately, throughout most of the discussion the subject is strictly adhered to, and any theory of explanation involving assumptions contradictory to the principles of physical science is considered irrevelant. The issue is, in the main, considered to be whether certain strange phenomena are explicable by what we know of conjuring, mal-observation, and the psychology of belief, or whether they must be

Proceedings of the English society for psychical research. Part x, Oct., 1886. London, Trübner. transferred to the category of the unexplained, to be reserved for future study. Can we say "yes" or ' no' to this alternative, and if not, which of these answers is pointed out as the more probable?

Professor Barrett opens the discussion, and at once records his conviction, "that, at any rate, some of the simpler phenomena of spiritualism are inexplicable by any causes at present recognized by science." This conviction is not due to experience gained in the ordinary public seances ; that is considered as largely fraudulent, and evidently worthless. But attention is called to a few cases of private mediumship, in which all the conditions necessary for a scientific test were granted. In the first of these cases, a girl of ten years had the peculiar gift of causing raps to be heard, even when her hands and feet were firmly held : the raps occurred at certain letters of the alphabet. and displayed a childish intelligence. A word "was misspelled by raps, exactly as the child would have misspelled that word." Professor Barrett concludes that he is ' morally certain' that hallucination, trickery, or known causes had nothing to do with it, but that it belongs to a 'class of phenomena wholly new to science.' A case is then cited in which a clever boy deceived his father (a distinguished surgeon) and all his family, by pretended spiritualistic manifestations, for a whole year ; but the ' radical' difference is pointed out that in this case the trick was discovered, in the former case it was not. Professor Barrett, with another private medium, saw tables move, and raps spell out 'pious platitudes,' "such as the medium herself (a Methodist) would be likely to concoct," and again considers the phenomena as inexplicable. A seance with a paid medium, Mr. Englinton, added to the mystery. Whether further study will support this conviction or not, at any rate, says Professor Barrett, more light can be shed on these phenomena by occasionally assuming the possibility of the spiritualistic stand-point; theorizing is needed as well as observation.

The paper of Mrs. Henry Sidgwick reports an unusually able investigation of the so-called spiritualistic phenomena. It is the account of an observer who knows how enormous the possibilities of deception, of mal-observation, and how insidiously inference usurps the place of perception. Every one interested in the psychology of illusions should read this admirable exposition of an interesting chapter on that subject.

The phenomena to be explained include raps, levitation of objects, playing on musical instruments, psycography and so on. Mrs. Sidgwick speaks from a twelve years' experience with mediums, including several of considerable fame. As 